July 1994

IFP-702-UNC

VAND-TH-94-8

\title{
Simple Non-Abelian Finite Flavor Groups and Fermion Masses
}

\author{
P. H. Frampton ${ }^{(a)}$ and T. W. Kephart ${ }^{(a, b)}$ \\ (a)Institute of Field Physics, Department of Physics and Astronomy, \\ University of North Carolina, Chapel Hill, NC 27599-3255 \\ (b)Department of Physics and Astronomy, Vanderbilt University, Nashville, TN 37235*
}

\begin{abstract}
The use of nonabelian discrete groups $G$ as family symmetries is discussed in detail. Out of all such groups up to order $\mathrm{g}=31$, the most appealing candidates are two subgroups of $\mathrm{SU}(2)$ : the dicyclic [double dihedral] group $\mathrm{G}=Q_{6}={ }^{(d)} D_{3}(\mathrm{~g}=12)$ and the double tetrahedral group ${ }^{(d)} T=Q_{4} \tilde{\times} Z_{3}$ $(\mathrm{g}=24)$. Both can allow a hierarchy $t>b, \tau>c>s, \mu>u, d, e$. The top quark is uniquely allowed to have a G symmetric mass. Sequential breaking of $\mathrm{G}$ and radiative corrections give the smaller masses. Anomaly freedom for gauging $G \subset S U(2)$ is a strong constraint in assignment of fermions to representations of $\mathrm{G}$.
\end{abstract}

Typeset using REVTEX

*Permanent address 


\section{Introduction}

To cite just a few of very many examples, discrete groups are central to molecular orbitals just as the crystallographic groups are in solid-state physics; the discrete symmetries $\mathrm{C}, \mathrm{P}$ and $\mathrm{T}$ and their violation have made a profound impact on our understanding of quantum field theory. Thus, finite groups play a major role in physics.

In the minimal standard model, nine of the nineteen parameters are quark and lepton masses, and four more pertain to quark flavor mixing. Thus more than $2 / 3$ of the parameters are associated with fermion masses. The fermion masses range from the electron mass $(0.511 \mathrm{MeV})$ up to the top quark mass $(174 \mathrm{GeV})$, a ratio of 340,000 . With a single Higgs doublet, this implies a corresponding puzzling hierarchy in the Yukawa coupling constants.

This hierarchy can be ameliorated by the postulation of a flavor or family symmetry group $\mathrm{G}$ underlying the mass pattern. The $\mathrm{G}$ we employ will be nonabelian because a nonabelian group has nontrivial multidimensional representations; $G$ will also be taken as finite because this allows more low dimensional representations and hence more structure than possible for a continuous Lie symmetry group. Nevertheless, it will be important that $\mathrm{G}$ be gaugeable to ensure consistency with gravity, and this will constrain the assignments of fermions to representations of $\mathrm{G}$.

The possible finite groups up to order $\mathrm{g}=31$ ( It is traditional to stop one below a power of 2 because of the proliferation of finite groups for each $g=2^{n}$ ) are less than one hundred in number. They are all listed in Section II below. The gauging of G is described in Section III.

Generalities of model building are in Section IV; specific examples, most notably $Q_{6}$ and ${ }^{(d)} T$ are considered in Section V. Finally, in Section VI are some concluding remarks. 


\section{Non-Abelian Finite Groups Up To Order 31}

From any good textbook on finite groups [1] we may find a tabulation of the number of finite groups as a function of the order $\mathrm{g}$, the number of elements in the group. Up to order 31 there is a total of 93 different finite groups of which slightly over one half (48) are abelian.

Amongst finite groups, the non-abelian examples have the advantage of non-singlet irreducible representations which can be used to inter-relate families. Which such group to select is based on simplicity: the minimum order and most economical use of representations [2].

Let us first dispense with the abelian groups. These are all made up from the basic unit $Z_{p}$, the order $\mathrm{p}$ group formed from the $p^{\text {th }}$ roots of unity. It is important to note that the the product $Z_{p} Z_{q}$ is identical to $Z_{p q}$ if and only if $\mathrm{p}$ and $\mathrm{q}$ have no common prime factor.

If we write the prime factorization of $g$ as:

$$
g=\prod_{i} p_{i}^{k_{i}}
$$

where the product is over primes, it follows that the number $N_{a}(g)$ of inequivalent abelian groups of order $\mathrm{g}$ is given by:

$$
N_{a}(g)=\prod_{k_{i}} P\left(k_{i}\right)
$$

where $P(x)$ is the number of unordered partitions of $x$. For example, for order $g=144=2^{4} 3^{2}$ the value would be $N_{a}(144)=P(4) P(2)=5 \times 2=10$. For $g \leq 31$ it is simple to evaluate $N_{a}(g)$ by inspection. $N_{a}(g)=1$ unless g contains a nontrivial power $\left(k_{i} \geq 2\right)$ of a prime.

These exceptions are: $N_{a}(g=4,9,12,18,20,25,28)=2 ; N_{a}(8,24,27)=3$; and $N_{a}(16)=5$. This confirms that:

$$
\sum_{g=1}^{31} N_{a}(g)=48
$$

We shall not consider these abelian cases further in this paper. 
Of the nonabelian finite groups, the best known are perhaps the permutation groups $S_{N}$ (with $N \geq 3$ ) of order $N$ ! The smallest non-abelian finite group is $S_{3}\left(\equiv D_{3}\right)$, the symmetry of an equilateral triangle with respect to all rotations in a three dimensional sense. This group initiates two infinite series, the $S_{N}$ and the $D_{N}$. Both have elementary geometrical significance since the symmetric permutation group $S_{N}$ is the symmetry of the N-plex in $\mathrm{N}$ dimensions while the dihedral group $D_{N}$ is the symmetry of the planar N-agon in 3 dimensions. As a family symmetry, the $S_{N}$ series becomes uninteresting rapidly as the order and the dimensions of the representions increase. Only $S_{3}$ and $S_{4}$ are of any interest as symmetries associated with the particle spectrum [4], also the order (number of elements) of the $S_{N}$ groups grow factorially with $\mathrm{N}$. The order of the dihedral groups increase only linearly with $\mathrm{N}$ and their irreducible representations are all one- and two- dimensional. This is reminiscent of the representations of the electroweak $S U(2)_{L}$ used in Nature.

Each $D_{N}$ is a subgroup of $O(3)$ and has a counterpart double dihedral group $Q_{2 N}$, of order $4 N$, which is a subgroup of the double covering $S U(2)$ of $O(3)$.

With only the use of $D_{N}, Q_{2 N}, S_{N}$ and the tetrahedral group T ( of order 12, the even permutations subgroup of $S_{4}$ ) we find 32 of the 45 nonabelian groups up to order 31, either as simple groups or as products of simple nonabelian groups with abelian groups: (Note that $D_{6} \simeq Z_{2} \times D_{3}, D_{10} \simeq Z_{2} \times D_{5}$ and $D_{14} \simeq Z_{2} \times D_{7}$ ) 


\begin{tabular}{||c||c||}
\hline $\mathrm{g}$ & \\
\hline 6 & $D_{3} \equiv S_{3}$ \\
\hline 8 & $D_{4}, Q=Q_{4}$ \\
\hline 10 & $D_{5}$ \\
\hline 12 & $D_{6}, Q_{6}, T$ \\
\hline 14 & $D_{7}$ \\
\hline 16 & $D_{8}, Q_{8}, Z_{2} \times D_{4}, Z_{2} \times Q$ \\
\hline 18 & $D_{9}, Z_{3} \times D_{3}$ \\
\hline 20 & $D_{10}, Q_{10}$ \\
\hline 22 & $D_{11}$ \\
\hline 24 & $D_{12}, Q_{12}, Z_{2} \times D_{6}, Z_{2} \times Q_{6}, Z_{2} \times T$, \\
\hline & $Z_{3} \times D_{4}, D_{3} \times Q, Z_{4} \times D_{3}, S_{4}$ \\
\hline 26 & $D_{13}$ \\
\hline 28 & $D_{14}, Q_{14}$ \\
\hline 30 & $D_{15}, D_{5} \times Z_{3}, D_{3} \times Z_{5}$ \\
\hline
\end{tabular}

There remain thirteen others formed by twisted products of abelian factors. Only certain such twistings are permissable, namely (completing all $g \leq 31$ )

\begin{tabular}{||c||c||}
\hline \multicolumn{1}{|c||}{} & \\
\hline 16 & $Z_{2} \tilde{\times} Z_{8}$ (two, excluding $\left.D_{8}\right), Z_{4} \tilde{\times} Z_{4}, Z_{2} \tilde{\times}\left(Z_{2} \times Z_{4}\right)$ (two) \\
\hline 18 & $Z_{2} \tilde{\times}\left(Z_{3} \times Z_{3}\right)$ \\
\hline 20 & $Z_{4} \tilde{\times} Z_{7}$ \\
\hline 21 & $Z_{3} \tilde{\times} Z_{7}$ \\
\hline 24 & $Z_{3} \tilde{\times} Q, Z_{3} \tilde{\times} Z_{8}, Z_{3} \tilde{\times} D_{4}$ \\
\hline 27 & $Z_{9} \tilde{\times} Z_{3}, Z_{3} \tilde{\times}\left(Z_{3} \times Z_{3}\right)$ \\
\hline
\end{tabular}

It can be shown that these thirteen exhaust the classification of all inequivalent finite groups up to order thirty-one [1]. 
Of the 45 nonabelian groups, the dihedrals $\left(D_{N}\right)$ and double dihedrals $\left(Q_{2 N}\right)$, of order $2 \mathrm{~N}$ and $4 \mathrm{~N}$ respectively, form the simplest sequences. In particular, they fall into subgroups of $O(3)$ and $S U(2)$ respectively, the two simplest nonabelian continuous groups.

For the $D_{N}$ and $Q_{2 N}$, the multiplication tables, as derivable from the character tables, are simple to express in general. $D_{N}$, for odd $\mathrm{N}$, has two singlet representations $1,1^{\prime}$ and $m=(N-1) / 2$ doublets $2_{(j)}(1 \leq j \leq m)$. The multiplication rules are:

$$
\begin{gathered}
1^{\prime} \times 1^{\prime}=1 ; \quad 1^{\prime} \times 2_{(j)}=2_{(j)} \\
2_{(i)} \times 2_{(j)}=\delta_{i j}\left(1+1^{\prime}\right)+2_{(\min [i+j, N-i-j])}+\left(1-\delta_{i j}\right) 2_{(|i-j|)}
\end{gathered}
$$

For even $\mathrm{N}, D_{N}$ has four singlets $1,1^{\prime}, 1^{\prime \prime}, 1^{\prime \prime \prime}$ and $(m-1)$ doublets $2_{(j)}(1 \leq j \leq m-$ 1)where $m=N / 2$ with multiplication rules:

$$
\begin{gathered}
1^{\prime} \times 1^{\prime}=1^{\prime \prime} \times 1^{\prime \prime}=1^{\prime \prime \prime} \times 1^{\prime \prime \prime}=1 \\
1^{\prime} \times 1^{\prime \prime}=1^{\prime \prime \prime} ; 1^{\prime \prime} \times 1^{\prime \prime \prime}=1^{\prime} ; 1^{\prime \prime \prime} \times 1^{\prime}=1^{\prime \prime} \\
1^{\prime} \times 2_{(j)}=2_{(j)} \\
1^{\prime \prime} \times 2_{(j)}=1^{\prime \prime \prime} \times 2_{(j)}=2_{(m-j)} \\
2_{(j)} \times 2_{(k)}=2_{|j-k|}+2_{(\min [j+k, N-j-k])}
\end{gathered}
$$

(if $k \neq j,(m-j))$

$$
2_{(j)} \times 2_{(j)}=2_{(\min [2 j, N-2 j])}+1+1^{\prime}
$$

(if $j \neq m / 2$ )

$$
2_{(j)} \times 2_{(m-j)}=2_{|m-2 j|}+1^{\prime \prime}+1^{\prime \prime \prime}
$$

(if $j \neq m / 2$ ) 


$$
2_{m / 2} \times 2_{m / 2}=1+1^{\prime}+1^{\prime \prime}+1^{\prime \prime \prime}
$$

This last is possible only if $\mathrm{m}$ is even and hence if $\mathrm{N}$ is divisible by four.

For $Q_{2 N}$, there are four singlets $1,1^{\prime}, 1^{\prime \prime}, 1^{\prime \prime \prime}$ and $(N-1)$ doublets $2_{(j)}(1 \leq j \leq(N-1))$. The singlets have the multiplication rules:

$$
\begin{gathered}
1 \times 1=1^{\prime} \times 1^{\prime}=1 \\
1^{\prime \prime} \times 1^{\prime \prime}=1^{\prime \prime \prime} \times 1^{\prime \prime \prime}=1^{\prime} \\
1^{\prime} \times 1^{\prime \prime}=1^{\prime \prime \prime} ; 1^{\prime \prime \prime} \times 1^{\prime}=1^{\prime \prime}
\end{gathered}
$$

for $N=(2 k+1)$ but are identical to those for $D_{N}$ when $\mathrm{N}=2 \mathrm{k}$.

The products involving the $2_{(j)}$ are identical to those given for $D_{N}(\mathrm{~N}$ even) above.

This completes the multiplication rules for 19 of the 45 groups. When needed, rules for the other groups will be derived. 


\section{Gauged Finite Groups and Anomalies}

The models we shall consider have a symmetry comprised of the standard model gauge group $S U(3)_{C} \times S U(2)_{L} \times U(1)_{Y}$ producted with a nonabelian finite group $\mathrm{G}$.

If $\mathrm{G}$ is a global (ungauged) symmetry, there are problems if the spacetime manifold is topologically nontrivial since it has been shown that any such global symmetry is broken in the presence of wormholes [5]. From a Local viewpoint (Local with a capital means within a flat spacetime neighbourhood) the distinction between a global and local (gauged) finite symmetry does not exist. The distinction exists only in a Global sense (Global meaning pertaining to topological aspects of the manifold). In a flat spacetime, gauging a finite group has no meaning. In the presence of wormholes, themselves expected to be inevitable from the fluctuations occurring in quantum gravity, gauging $\mathrm{G}$ is essential. The mathematical treatment of such a gauged finite group has a long history [6].

In order to gauge the finite group $\mathrm{G}$, the simplest procedure is to gauge a continuous group $\mathrm{H}$ which contains $\mathrm{G}$ as a subgroup, and then to spontaneously break $\mathrm{H}$ by choice of a Higgs potential. The symmetry breaking may occur at a high energy scale, and then the low energy effective theory will not contain any gauge potentials or gauge bosons; this effective theory is, as explained above, Locally identical to a globally-invariant theory with symmetry G.

For example, consider $\mathrm{G}=Q_{6}$ and $\mathrm{H}=S U(2)$. We would like to use only one irreducible representation $\Phi$ of $Q_{6}$ in the symmetry-breaking potential $V(\Phi)$. The irreps. of $Q_{6}$ are $1,1^{\prime}, 1^{\prime \prime}, 1^{\prime \prime \prime}, 2,2_{S}$. The $1^{\prime \prime}, 1^{\prime \prime \prime}$ and $2_{S}$ are spinorial and appear in the decompositions only of $2,4,6,8 \ldots$ of $S U(2)$. Since $\Phi$ must contain the 1 of $Q_{6}$ we must choose from the vectorial irreps. $3,5,7,9 \ldots$ of $S U(2)$. The appropriate choice is the 7 represented by a symmetric traceless third-rank tensor $\Phi_{i j k}$ with $\Phi_{i k k}=0$.

For the vacuum expectation value, we choose

$$
<\Phi_{111}>=+1 ;<\Phi_{122}>=-1
$$


and all other unrelated components vanishing. If we look for the $3 \times 3$ matrices $R_{i j}$ whicg leave invariant this VEV we find from choices of indices in

$$
R_{i l} R_{j m} R_{k n}<\Phi_{l m n}>=<\Phi_{i j k}>
$$

that $R_{31}=R_{32}=0\left(\mathrm{Use}<\Phi_{3 i j} \Phi_{3 i j}>=0\right)$ and that $R_{33}= \pm 1$. Then we find $\left(R_{11}\right)^{3}-$ $3 R_{11}\left(R_{12}\right)^{2}=1$ (Use $l=m=n=1$ in $(18)$ ). This means that if $R_{11}=\cos \theta$ then $\cos 3 \theta=1$ or $\theta_{n}=2 \pi n / 3$. So the elements of $Q_{6}$ are $A=R_{3}\left(\theta_{1}\right), A^{2}, A^{3}$ and $B, B A, B A^{2}$ where $B=$ $\operatorname{diag}(i,-i-i)$.

More generally, it can be shown that to obtain $Q_{2 N}$ one must use an $N^{\text {th }}$ rank tensor because one finds for the elements $R_{11}$ and $R_{12}$ :

$$
\sum_{p=0}^{[N / 2]}(-1)^{p}\left(\begin{array}{l}
N \\
2 p
\end{array}\right)\left(R_{11}\right)^{N-2 p}\left(R_{12}\right)^{2 p}=\cos N \theta=1
$$

If the group $\mathrm{H}$ is gauged, it must be free from anomalies. This entails several conditions which must be met:

(a) The chiral fermions must fall into complete irreducible representations not only of G but also of $\mathrm{H}$.

(b) These representations must be free of all $\mathrm{H}$ anomalies including $(H)^{3},(H)^{2} Y$; for the cases of $\mathrm{H}=O(3), S U(2)$ only the latter anomaly is nontrivial.

(c) If $\mathrm{H}=S U(2)$, there must be no global anomaly.

The above three conditions apply to nonabelian $\mathrm{H}$. The case of an abelian $\mathrm{H}$ avoids (a) and (c) but gives rise to additional mixed anomalies in (b).

For nonabelian H, conditions (b) and (c) are straightforward to write down and solve. Condition (a) needs more discussion. We shall focus on the special cases of $O(3) \supset D_{N}$ and $S U(2) \supset Q_{2 N}$

For $O(3)$ the irreps. are $\mathbf{1}, \mathbf{3}, \mathbf{5}, \mathbf{7}, \ldots$ dimensional. $D_{N}$ has irreps. (for even $N=2 m$ ) $1,1^{\prime}, 1^{\prime \prime}, 1^{\prime \prime \prime}$ and $2_{(j)}(1 \leq j \leq(m-1))$ and these correspond to:

$$
O(3): \mathbf{1} \rightarrow 1 ; \mathbf{3} \rightarrow\left(1^{\prime}+2_{(1)}\right)
$$


The same situation occurs for odd $\mathrm{N}$ with irreps. $1,1^{\prime}$ and $2_{(j)}(1 \leq j \leq(N-1) / 2)$. If we insist on keeping within the fermions of the standard model, or as close to that ideal as possible, nothing beyond a $\mathbf{3}$ is necessary because the same quantum numbers are not repeated more than three times.

For $S U(2) \supset Q_{2 N}$ the corresponding breakdown is:

$$
\mathbf{1} \rightarrow 1 ; \mathbf{2} \rightarrow 2_{S(1)} ; \mathbf{3} \rightarrow 1^{\prime}+2_{(1)}
$$

where the doublets of $Q_{2 N}, 2_{(1)}$ and $2_{S(1)}$, are defined by Eq. (20).

These are the principal splittings of a continuous group irrep. into finite subgroup irreps. we shall need in our discussions of model building below. 


\section{Model Building in General}

In order to be specific we need to set up a collection of model-building rules. The main purpose is to understand why the third family of quarks and leptons is heavy, and especially why the top quark is very heavy. Thus we require that:

(A) The t quark mass (and only the t ) transforms as a $\mathbf{1}$ of $\mathrm{G}$.

(B) The b and $\tau$ masses appear as $\mathrm{G}$ is broken to $G^{\prime}$.

We next require that at tree level or one-loop level the second family be distinguishable from the first. That is:

(C) After stage (B) first the c mass $\left(G^{\prime} \rightarrow G^{\prime \prime}\right)$, then the s and $\mu$ masses $\left(G^{\prime \prime} \rightarrow G^{\prime \prime \prime}\right)$ are generated.

At stage $(\mathrm{C})$ the $\mathrm{u}, \mathrm{d}$ and e remain massless.

Inaddition to the above constraints we require that:

(D) No additional quarks and a minimal number of leptons be introduced beyond the usual three-family standard model.

(E) All anomalies are cancelled as described in Section III above, when G is embedded in the minimal continuous Lie group $\mathrm{H}: G \subset H$.

We strive to satisfy all of (A) through (E). By the study of specific cases in the following Section $\mathrm{V}$ we shall see that these constraints are quite nontrivial to satisfy simultaneously and that the number of interesting models is small (We shall arrive at only two). 


\section{Specific Examples}

We shall treat special cases for the nonabelian 8] group $\mathrm{G}$ in turn, taken from the complete listing, up to order $\mathrm{g}=31$, given in Section II above.

(a) Dihedral Groups $\left(D_{N}\right.$, order $\left.g=2 N\right)$

From the multiplication rules for $D_{N}$ we see that if the top quark mass transforms as a singlet, as required by rule $(\mathrm{A})$ above, the $t_{L}$ and $t_{R}$ must both be in 1 or $1^{\prime}$ or the same $2_{(j)}$. The doublet is unsuitable because it will include a second quark, violating rule (A).

To proceed systematically, note that there are three triples of quarks with common quantum numbers: $(1)(t, b)_{L},(c, s)_{L},(u, d)_{L} ;(2) t_{R}, c_{R}, u_{R}$; and $(3) b_{R}, s_{R}, d_{R}$. Since $D_{N}$ is a subgroup of $S O(3)$ we must look to the rule (E) to see that each triple must be in $1+1+1$ or $1^{\prime}+2_{(1)}$ (equivalent to the vector 3 of $O(3)$ as can be deduced from the $O(3)$ and $D_{N}$ character tables) of $D_{N}$ to avoid anomalies. If $t_{L}$ and $t_{R}$ are in $1^{\prime}$ it follows that $(c, s)_{L}$, $(u, d)_{L}$ and $c_{R}, u_{R}$ are in $2_{(1)}$ implying that charm and up quarks have singlet components

in their mass terms, hence violating rule $(\mathrm{A})$. If $t_{L}$ and $t_{R}$ are in 1 , then so are $(c . s)_{L},(u, d)_{L}$ and $c_{R}, u_{R}$ again violating rule $(\mathrm{A})$.

From this discussion we deduce that no suitable model based on $\mathrm{G}=D_{N}$ exists.

(b) Permutation Groups $\left(S_{N}\right.$, order $g=N$ !)

The group $S_{3}$ is identical to $D_{3}$ which was excluded in (a) above. The only other $S_{N}$ with $g \leq 31$ is $S_{4}$ which has irreducible representations $1,1^{\prime}, 2,3,3^{\prime}$. It is a subgroup of $O(3)$ so the triples must be in $1+1+1$ or $3\left(5\right.$ of $\left.O(3) \rightarrow 2+3^{\prime}\right)$. Since $3 \times 3=1+2+3+3^{\prime}$ contains a singlet, neither choice fulfils rule (A).

Hence the groups $\mathrm{G}=S_{N}$ are excluded. 
(c) Tetrahedral Group (T, order $g=12)$

The group $\mathrm{T}$ has $1,1^{\prime}, 1^{\prime \prime}, 3$ representations and $T \subset O(3)$ with irreps. of $O(3)$ decomposing under $\mathrm{T}$ as $1 \rightarrow 1,3 \rightarrow 3,5 \rightarrow 1^{\prime}+1^{\prime \prime}+3$. Thus $t_{L}$ and $t_{R}$ must either both be in 1 or 3 and since $3 \times 3=1+1^{\prime}+1^{\prime \prime}+2(3)$ both choices violate rule $(\mathrm{A})$.

Hence T cannot be used.

(d) Double Dihedral (or Dicyclic) Groups $\left(Q_{2 N}\right.$, order $g=4 N$ )

The above cases (a),(b),(c) are all subgroups of $O(3)$. There exist counterparts which are doubled and are subgroups not of $O(3)$ but of $S U(2)$.

As a first example, consider ${ }^{(d)} D_{N}=Q_{2 N}$ which has representations $1,1^{\prime}, 1^{\prime \prime}, 1^{\prime \prime \prime}$ and $(N-1)$ doublets $2_{(j)}$ as described above in Section II. In $S U(2)$ the representations decompose under $Q_{2 N}$ as: $1 \rightarrow 1,2 \rightarrow 2_{(2)}$ and $3 \rightarrow 1^{\prime}+2_{(1)}$. Thus the possible choices for the three quark triples are: $1+1+1,1+2_{(2)}$ and $1^{\prime}+2_{(1)}$.

We can now go a long way toward fulfilling all the rules in Section IV above.

In the quark sector $t_{L}$ and $t_{R}$ must be both 1 or both $1^{\prime}$. The latter leads to other singlet mass terms from $2_{(1)} \times 2_{(1)}$ and so is excluded. Thus $t_{L}$ and $t_{R}$ must both be 1 . The simplest choice is then to use $Q_{6}\left(Q_{2 N}, N \geq 4\right.$ leads to no new structure) and then there are two different assignments within $Q_{6}$, namely:

Choice A. 


$$
\begin{aligned}
& \left(\begin{array}{l}
t \\
b
\end{array}\right)_{L} \begin{array}{llll}
1 & t_{R} & 1 \\
& & b_{R} & 1^{\prime}
\end{array}\left(\begin{array}{c}
\nu_{\tau} \\
\tau
\end{array}\right)_{L} \begin{array}{llll}
1 & \tau_{R} & 1^{\prime}
\end{array}
\end{aligned}
$$

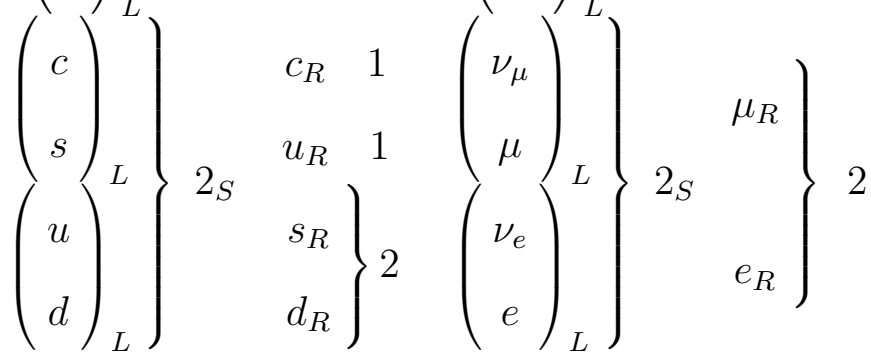

Choice B.

$$
\begin{aligned}
& \left(\begin{array}{l}
t \\
b
\end{array}\right)_{L} \begin{array}{llll}
1 & t_{R} & 1 \\
& & b_{R} & 1^{\prime}
\end{array} \quad\left(\begin{array}{c}
\nu_{\tau} \\
\tau
\end{array}\right)_{L} \begin{array}{llll}
1 & \tau_{R} & 1^{\prime}
\end{array}
\end{aligned}
$$

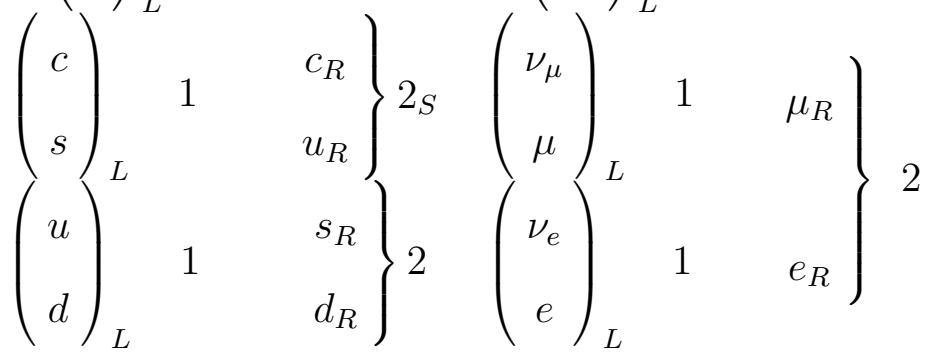

Both choices A and B are free from $\left(S U(2)^{\prime}\right)^{3}$ anomalies. Now consider the mixed $\left(S U(2)^{\prime}\right)^{2} Y$ anomaly. It is nonvanishing for both A and B. This is inevitable for any assignment even for the case of $Q_{6}$ as can be seen by studying the following table. Normalize the quadratic Casimir of the $S U(2)^{\prime}$ doublet to +1 and hence the triplet to +4 ; define $Q=T_{3}+Y$ and the anomaly is: 


\begin{tabular}{||c||c|c|c||}
\hline & $2_{S}+1$ & 3 & $1+1+1$ \\
\hline \hline$\left(\begin{array}{c}\nu \\
e^{-}\end{array}\right)_{L i}$ & -1 & -4 & 0 \\
$e_{L i}^{+}$ & +1 & +4 & 0 \\
\hline$\left(\begin{array}{c}u \\
d\end{array}\right)_{L i}$ & +1 & +4 & 0 \\
$\bar{u}_{L i}$ & -2 & -8 & 0 \\
$\bar{d}_{L i}$ & +1 & +4 & 0 \\
\hline
\end{tabular}

Thus this anomaly adds to +8 for Choice A, +6 for Choice B. To cancel this $\mathrm{n}$ the most economial waye, can add appropriate additional leptons. From the above Table, we see that for all choices of $Q_{6}$ representations we need to extend slightly the lepton sector whereupon cancellation of all anomalies is always possible.

For example, in choice $\mathrm{A}$ the anomaly +8 can be compensated by adding two $3 \mathrm{~s}$ of $S U(2)^{\prime}$ of left-handed leptons and corresponding singlets of right-handed leptons with usual quantum numbers. The quark sector is exactly as in the standard model. The additional particles might be called Q-leptons. Because their masses break $S U(2)_{L}$, their masses should be below about $200 \mathrm{GeV}$; but phenomenology dictates that they be above $50 \mathrm{GeV}$.

For the Choice A the mass matrices are:

$$
U=\left(\begin{array}{c|c}
<2_{S}> & <2_{S}> \\
\hline<1> & <1>
\end{array}\right)
$$

and: 


$$
D=L=\left(\begin{array}{c|c}
<1^{\prime \prime}+1^{\prime \prime \prime}+2_{S}> & <2_{S}> \\
\hline<2> & <1^{\prime}>
\end{array}\right)
$$

The way of implementing the hierarchy is by the following steps which comply with the rules (A) - (D) of Section IV above:

(A) A VEV to an $S U(2)_{L}$ doublet which is a singlet of $Q_{6}$ gives $t$ its heavy mass without breaking $Q_{6}$.

(B) A VEV to a $1^{\prime}$ gives $\mathrm{b}$ and $\tau$ their masses, at the same time breaking $G=Q_{6}$ to $G^{\prime}=Z_{6}$

(C) and (D) The charm quark mass is radiatively generated according to the diagram of Fig. (1) which uses a VEV transforming as $\left(1,2_{S}\right)$ under $S U(2)_{L} \times S U(2)^{\prime}$. Next the s and $\mu$ acquire their tree-level masses through a $\left(2,1^{\prime \prime}\right.$ or $\left.1^{\prime \prime \prime}\right)$ VEV. These VEVs break $Z_{6}$ completely. At this point the $\mathrm{u}, \mathrm{d}$ and e are still massless.

(e) Double Tetrahedral Group $\left({ }^{(d)}\right.$ T, order $\left.g=24\right)$

The doubled group ${ }^{(d)} T \subset S U(2)^{\prime}$ has representations $1,1^{\prime}, 1^{\prime \prime}, 2_{S}, 2_{S}^{\prime}, 2_{S}^{\prime \prime}$ and 3 . The lowest dimensional representations of $S U(2)^{\prime}$ decompose as: $1 \rightarrow 1,2 \rightarrow 2_{S}, 3 \rightarrow 3$. This leads to a model quite analogous to the $Q_{6}$ model described above with the same advantages. Note that ${ }^{(d)} T$ is isomorphic to $Z_{3} \tilde{\times} Q_{4}$. We assign:

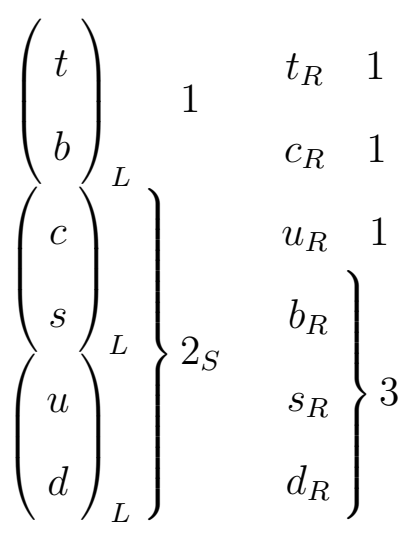




$$
\begin{aligned}
& \left.\left.\left(\begin{array}{c}
\nu_{\tau} \\
\tau
\end{array}\right) \begin{array}{l}
1 \\
\left(\begin{array}{c}
\nu_{\mu} \\
\mu
\end{array}\right)_{L} \\
\left(\begin{array}{c}
\nu_{e} \\
e
\end{array}\right)_{L}
\end{array}\right\} \begin{array}{rr}
2_{S} & \mu_{R} \\
& e_{R}
\end{array}\right\} 3
\end{aligned}
$$

whereupon the mass matrices are:

$$
U=\left(\begin{array}{c|c}
<2_{S}>\mid<1> \\
\hline<2_{S}>\mid<1>
\end{array}\right)
$$

and:

$$
D=L=\left(\begin{array}{c|c}
<2_{S}+2_{S}^{\prime}+2_{S}^{\prime \prime}> & <3> \\
\hline<2_{S}+2_{S}^{\prime}+2_{S}^{\prime \prime}> & <3>
\end{array}\right)
$$

To implement the hierarchy complying with rules (A) to (D) of Section IV gives:

(A) A VEV to a $S U(2)_{L}$ doublet which is a singlet of ${ }^{(d)} T$ gives a heavy mass to $t$ without breaking ${ }^{(d)} T$.

(B) A VEV to a 3 of ${ }^{(d)} T$ gives mass to $\mathrm{b}$ and $\tau$.

(C) and (D) The c quark acquires mass radiatively through a VEV of $\left(1,2_{S}\right)$ via the diagram of Fig. (1). The s and $\mu$ acquire mass at tree level through $2_{S}^{\prime}$ or $2_{S}^{\prime \prime}$ VEVs, breaking 
$G^{\prime}$. The $\mathrm{u}, \mathrm{d}$ and e are still massless.

(f) Other Groups

We have so far fealt with 22 of the 45 nonabelian groups with $g \leq 31$. Another 11 are not simple so fall outside our search. The remaining 12 are twisted products of $Z_{N}$ 's and of orders: $\mathrm{g}=16(5), 18,20,21,24(2)$ and $27(2)$. Note that one of these has already been discussed at length since ${ }^{(d)} T=Z_{3} \tilde{\times} Q_{4}$.

Of the remainder, $Z_{9} \tilde{\times} Z_{3}=\Delta(27)$, a subgroup of $S U(3)$. None of the others is embeddable in an $S U(2)^{\prime}$, since all such groups are considered in (a) -(e) above. The only other one which embeds in $S U(3)$ is $\Delta(24)=T \times Z_{2}$ but triplets do not allow a hierarchy following our rules of Section IV. The only other Lie group of interest with irreducible representations $\leq 3$ is $S O(4)=S U(2) \times S U(2)$ with a simple direct product. None of our list embeds minimally in $S O(4)$ because their products are twisted, and not simple.

Thus only two possibilities - $Q_{2 N}(N \geq 3)$ and ${ }^{(d)} T$ - permit a fermion hierarchy of the type we have specified. 


\section{Concluding Remarks}

We have exhibited models with a nonabelian discrete flavor group $G$ where $G$ can be embedded in an anomaly-free $S U(2)$. The top quark can be much heavier than all other quark flavors because it alone has a G-invariant mass.

The breaking of $\mathrm{G}$ gives rise sequentially to the other fermion masses: first $\mathrm{b}$ and $\tau$; then $\mathrm{c}$; and finally s and $\mu$. The first family masses are so tiny that they are neglected at the order considered here. For the simplest case of $G=Q_{6}$, a testable prediction is the occurrence of the Q-leptons in the mass range $50 \mathrm{GeV}$ to $200 \mathrm{GeV}$.

We should mention the point that the spontaneous breaking of discrete symmetries always gives rise to the danger of unacceptable cosmological domain walls [9]. However this danger can always be avoided by adding explicit soft breaking in the potential function.

Many outstanding questions remain such as: Can this scheme be made more quantitative? How would supersymmetry effect the model building? How does G arise in a more complete framework [10]?

Although we have removed the extreme hierarchy of the Yukawa couplings, it has been replaced by a more involved Higgs sector. This seems inevitable; the point is that the discrete group $\mathrm{G}$ leads to a new viewpoint that provides a first step to understanding the mass spectrum of quarks and leptons, particularly why the top quark mass is so different from all other fermion masses.

One of us (T.W.K.) thanks the members of the Institute of Field Physics at UNC-Chapel Hill for their generous hospitality while this work was in progress. This work was supported in part by the U.S. Department of Energy under Grants DE-FG05-85ER-40219 and DEFG05-85ER-40226. 


\section{REFERENCES}

[1] Useful sources of information on the finite groups include:

D. E. Littlewood, The Theory of Group Characters and Matrix Repesentations of Groups, Oxford (1940).

M. Hamermesh, Group Theory and Its Applications to Physical Problems, Addison-Wesley (1962).

J. S. Lomont, Applications of Finite Groups, Academic Press (1959), reprinted by Dover (1993).

A. D Thomas and G. V. Wood, Group Tables, Shiva Publishing (1980).

[2] Another (non-minimal by the above definition) route to discrete family symmetry is through the previous $S U(N)$ family symmetry models [3] . Here one can consider the breaking pattern

$$
S U(N) \rightarrow S U(5) \times S U(5-N) \times U(1) \rightarrow S U(5) \times G
$$

where $\mathrm{G}$ is a discrete group. Such models typically have more than just three complete families until $G$ is broken. Although worthy of further study, these models do not in general satisfy our minimality condition on the fermion spectrum.

[3] P. H. Frampton, Phys. Lett. 88B, 299 (1979);

H. Georgi, Nucl. Phys. B156, 126 (1979).

[4] S. Pakvasa and H. Sugawara, Phys. Lett. 73B, 61 (1978); ibid 82B, 105 (1979);

T. Brown, N. Deshpande, S. Pakvasa and H. Sugawara, ibid 141B, 95 (1984);

T. Brown, S. Pakvasa, H. Sugawara and Y. Yamanaka, Phys. Rev. D30, 255 (1984).

[5] S. Giddings and A. Strominger, Nucl. Phys. B307, 854 (1988);

S. Coleman, ibid B310, 643 (1988);

G. Gilbert, ibid B328. 159 (1989);

R. Holman, S. D. H. Hsu, T. W. Kephart, E. W. Kolb, R. Watkins and L. M. Widrow, 
Phys. Lett. B282, 132 (1992);

M. Kamionkowski and J. March-Russell, ibid 282, 137 (1992);

S. M. Barr and D. Seckel, Phys. Rev. D46, 539 (1992).

[6] "Invariants of Flat Bundles" by J. Cheeger, Proceedings of the International Congress of Mathematicians (Vancouver, 1974) "Multiplication of Differential Characters" by J. Cheeger, Instituto Nazionale di Alta Matematica, Symposia Mathematica, Volume XI (1973) "Differential Characters and Geometric Invariants" by J. Cheeger and J. Simons, A.M.S. Summer Institute on Differential Geometry (Lecture Notes), Stanford 1973. J. D. Stasheff, Private Communication.

[7] Note that even though N 3-vector VEVs are needed to break $S O(3)$ to $D_{N}$ the top Yukawa has a factor $N^{-1 / 2}$ suppression as can be seen by comparing the $\mathrm{t}$ and $\mathrm{W}$ masses.

[8] This is a generalization to non-abelian symmetry of:

L. Ibanez and G. G. Ross, Phys. Lett. B260, 291 (1991); Nucl. Phys. B368, 3 (1992).

[9] Ya. B. Zel'dovich, I. Yu. Kobzarev and L. B. Okun, Sov. Phys. JETP 40, 1 (1975).

[10] It is interesting to note that discrete groups are a natural outcome of Calabi-Yau and orbifold compactification of the heterotic $E_{8} \times E_{8}$ superstring [11]. There the maximal discrete groups are apparently subgroups of $S U(3)$ [12]. Discrete subgroups of $S U(3)$ have recently been considered as family groups by D. B. Kaplan and M. Schmaltz, Phys. Rev. D49, 3741 (1994).

[11] G. Lazarides and Q. Shafi, J. Math. Phys. 30, 711 (1989).

E. Zaslow, Nucl. Phys. B415, 155 (1994).

[12] W. M. Fairbairn, T. Fulton and W. H. Klink, J. Math. Phys. 5, 1038 (1964). 


\section{Figure Caption}

Fig. 1 One loop diagram contributing to the charm quark mass. 


\section{FIGURES}

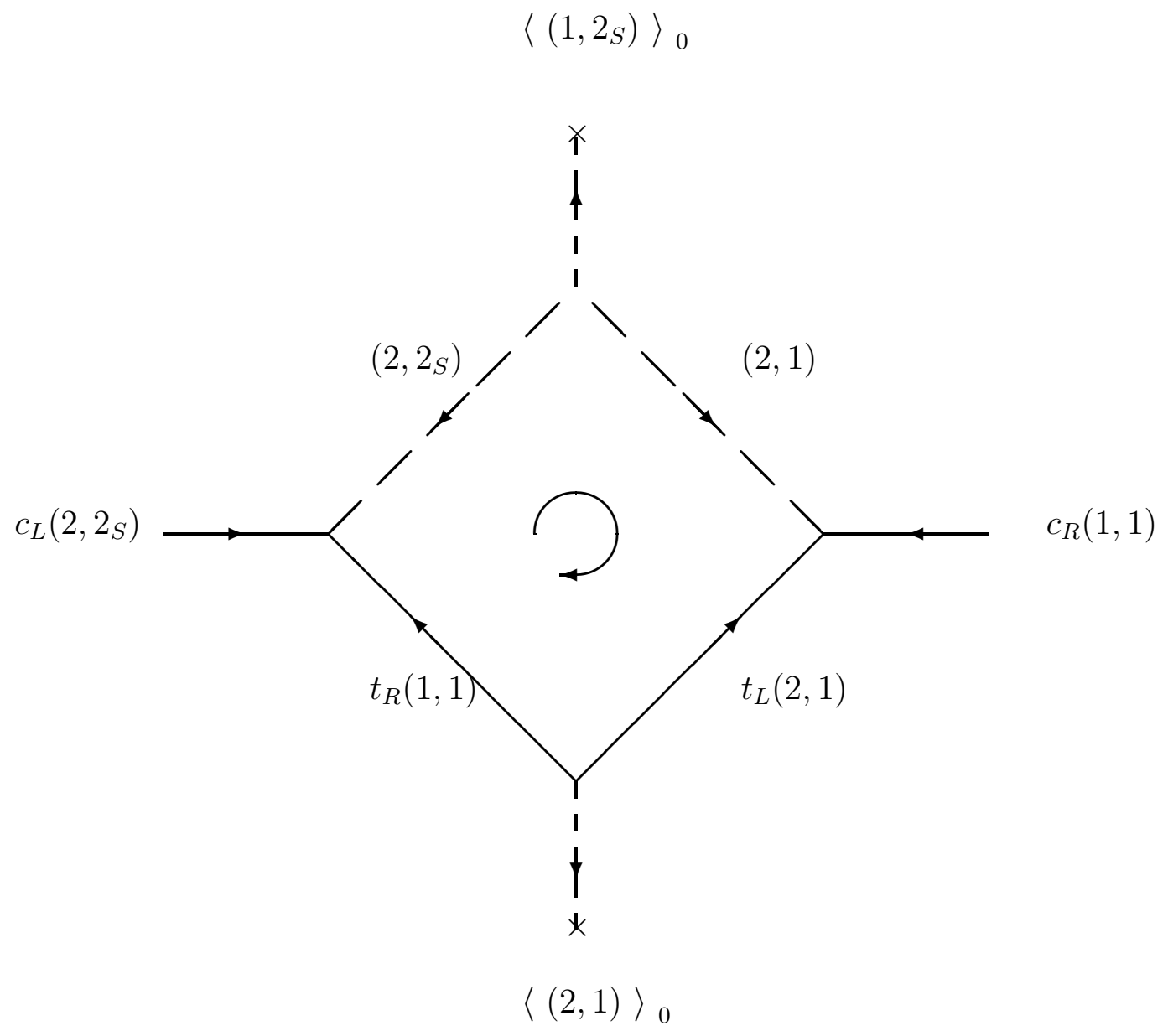

FIG. 1. One loop diagram contributing to the charm quark mass. 